\title{
Analysis of the performance of coded and uncoded mixed RF and multihop coherent OFDM-FSO systems for 5G-CRAN applications
}

\author{
${ }^{1}$ K. Anbarasi, R.G.Sangeetha*, C.Hemanth \\ School of Electronics Engineering, Vellore Institute of Technology, Chennai campus, Tamilnadu, India.

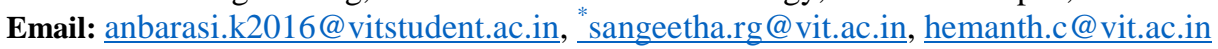

\begin{abstract}
The performance of coded and uncoded mixed single-hop RF links and multihop FSO link systems for the $5 \mathrm{G}$ centralized radio access network (C-RAN) is investigated in this paper using a coherent detection technique. A generalized Nakagami-m fading channel is chosen for the RF link, and the Gamma-Gamma distribution channel is chosen for the FSO link. The RF and FSO links use M-Quadrature Amplitude Modulated Orthogonal Frequency Division Multiplexing (M-QAM OFDM) modulation for the coherent detection scheme. The Meijer-G function is used to calculate outage probability (OP) and average symbol error rate (ASER) for analyzing the performance of coded and uncoded systems. Reed Solomon (RS) and Bose-Chaudhuri-Hocquenghem $(\mathrm{BCH})$ codes are used in the coded system. In relays, the decode and forward (DF) method is used. For analysis, the effects of atmospheric turbulence, atmospheric loss, and pointing error are considered. The results show that the coding technique improves the system's performance. When compared to $\mathrm{BCH}$ codes, $\mathrm{RS}$ codes improve system performance more. A Monte-Carlo simulation is used to validate the analytical results.
\end{abstract}

Index Terms: Average symbol Error Rate, Coherent, Multi-hop, coding technique, turbulence.

\section{Introduction}

\subsection{Background and Motivation}

An increase in mobile and internet access usage leads the emerging $5 \mathrm{G}$ network to meet the demands like high data rate, less power consumption, and less latency (Iovanna, Paola, et al. 2016). The Cloud / Centralized Radio Access Network (C-RAN) architecture is incorporated in the 5G network to meet the requirements. CRAN architecture consists of Baseband Unit (BBU) pool and Remote Radio Heads (RRH) (Zhang, Haijun, et 
al. 2016). RRH receives the radio signal from the user equipment (UE) and forwards it to the BBU pool, where the baseband signal processing occurs. BBU pool acts as a central processor that performs functions like modulation, coding, radio resource control, etc. 5G C-RAN requires reliable and high-speed links for front hauling between RRH and BBU pool (Ranaweera, Chathurika, et al., 2017).

Optical fiber and free-space optical (FSO) links give a solution to the front haul links for 5G C-RAN architecture to meet high capacity requirements. The deployment of optical fiber is challenging and costeffective. FSO is easy to deploy, which acts as a suitable front haul link for 5G networks (Ahmed et al., 2018). In C-RAN architecture, the signal from UE to RRH is a radio signal, and from RRH to BBU pool, it is the FSO front haul link. The structure of the architecture is like a dual-hop mixed RF-FSO system. Many works have been done in a dual-hop hybrid RF-FSO system.

In (E. Lee et al. 2011), a dual-hop mixed RF-FSO system with amplitude and forward (AF) technique is introduced with RF link as Rayleigh fading and FSO as Gamma-Gamma distribution without considering the pointing error. In (I. S. Ansari et al. 2013), the pointing error impact is considered for the same model in (E. Lee et al., 2011). Further extension of (I. S. Ansari et al. 2013) [6] is carried out in (E. Zedini et al. 2015), in which generalized Nakagami-m distribution is considered as RF link, and the analysis is performed in both heterodyne and IM/DD detection techniques. The advantage of the decode and forward (DF) technique over AF is discussed in (Anees, S, and M. R. Bhatnagar 2015) for the same model in (E. Zedini et al. 2015). DF technique with $\eta-\mu$ RF link, which includes Nakagami-m fading channel as a particular case, and FSO with gamma-gamma distribution for the two detection techniques, is considered in (Sharma et al. 2016). In C-RAN architecture, under fixed BBU pool location, the link availability can be obtained by connecting serial relays between RRH and BBU pool. Long link distance can be covered by using short links using relays. RF link between UE and RRH cannot be increased as the RF link suffers from power loss due to its high frequency. Multihop relaying of the FSO system overcomes the problem of expanding the coverage area. The multihop FSO system is studied in many papers (Akella et al., 2005). 
In (Akella et al. 2005) Multi-hop FSO system with the DF technique is studied and compared with AF relaying strategy. DF relaying performs better than AF relaying. In (Tang, Xuan, et al. 2014), heterodyne detection with differential phase-shift keying (DPSK) modulation scheme with Gamma-Gamma distribution is investigated for different atmospheric turbulence and pointing error conditions. In (Bekkali, Abdelmoula, et al. 2010), a coherent multihop method with the DF technique is implemented to obtain a better spatial selectivity property. Though a coherent system is complex to implement, its advantages over non-coherent systems are distinct. The coherent system is considered in this paper. Orthogonal frequency division multiplexing (OFDM) is recently adopted in many types of research for its advantages like high transmission rates of data, inter-symbol interference suppression, random fading effect prevention. In (Bekkali, Abdelmoula, et al. 2010), OFDM modulation with IM/DD technique for Gamma-Gamma distribution is discussed. Multilevel DPSK modulated non-equalization OFDM system with a coherent detection system is presented in Chen et al. 2014. In (Wang et al. 2015), quadrature amplitude modulated OFDM with tunable optical coherent detection is analyzed. Extension of (Wang et al. 2015) is given in (Wang et al. 2016), where a multihop coherent OFDM FSO system is implemented with an M-QAM modulation scheme with DF relaying technique with gamma-gamma distribution.

Among many other mitigation techniques like diversity, modulation schemes, etc. coding technique is considered to improve the system's performance. Reed Solomon (RS) and Bose-Chaudhuri-Hocquenghem $(\mathrm{BCH})$ codes are taken as mitigation techniques for the system model presented in this paper. The effect of these codes on the proposed system is analyzed in terms of average symbol error (ASER). Forward Error Correction codes add redundant bits to the information bits to overcome the error rates (Gupta, Nancy, et al., 2019). RS codes in high-speed, long-distance transmission are suitable for correcting burst errors and random bit errors (Yu, Meng, et al., 2004). BCH codes correct multiple bit errors in a codeword (Nayak, Anuj 2014). 


\subsection{Contribution}

Significant contributions in this paper are,

- A coherent detection method is used to evaluate the performance of mixed RF and multihop FSO systems.

- For the first time, RS $(15,11)$ and $\mathrm{BCH}(15,11)$ coding techniques for mixed RF and multihop FSO systems are introduced, and performance is compared for both coded and uncoded cases.

- For coherent detection, both RF and FSO links used M-QAM modulated OFDM modulation. The system's performance is evaluated under various turbulence, weather, and pointing errors conditions.

In the proposed work, we consider a single RF link to be a Generalized Nakagami-m fading channel as it includes the Nakagami-m fading channel as a particular case that covers the line of sight (LOS) component (Zedini Emna, Imran Shafique Ansari, et al. 2015). Generalized Nakagami-m fading channel is modeled for fading effects like multipath and shadow (E. Zedini et al. 2016). In C-RAN architecture, the distance between the UE and RRH is less, which also needs to consider LOS communication. A multihop FSO link is regarded as a Gamma-Gamma distributed channel. Experimental and simulation data fits all turbulence conditions for Gamma-Gamma distribution in point detectors than Log-Normal distribution (Wayne, David T., et al. 2010).

The rest of the paper is organized as follows. In section 2, the system and channel model is discussed, in section 3, Error Correcting Codes are presented, in section 4, average symbol error rate (ASER) is derived for the un-coded and coded system, in section 5, OP is derived for the uncoded system, in section 6 results and discussion is given. Finally, section 7 concludes the paper.

\section{System and Channel Model}

\subsection{System model}

The model of mixed RF and multihop FSO systems for the C-RAN architecture is shown in Fig. 1. In Fig. 1, the source node is the user equipment (UE), and it is denoted as source and destination is the centralized baseband unit (BBU), and it is represented as a destination, and the intermediate relay nodes are designated 
as a Relay. The number of hops is L, and the number of links between source and destination is L-1. The first node from source to RRH unit, i.e., Relay 1, is connected using the RF link, and, from i+1 to L hops, communication occurs through the FSO link. The relays follow the decode and forward (DF) technique in which the signal from the transmitter is transmitted to the relays, which demodulates, decodes, and retransmit the received signal after modulating it again to reduce the error, by this way, it defers from amplifying and forward technique in which the signal is amplified with noise and transmitted in each node. In a multihop transmission system, the error is reduced using the DF technique, which helps extend the transmission link distance of the system.

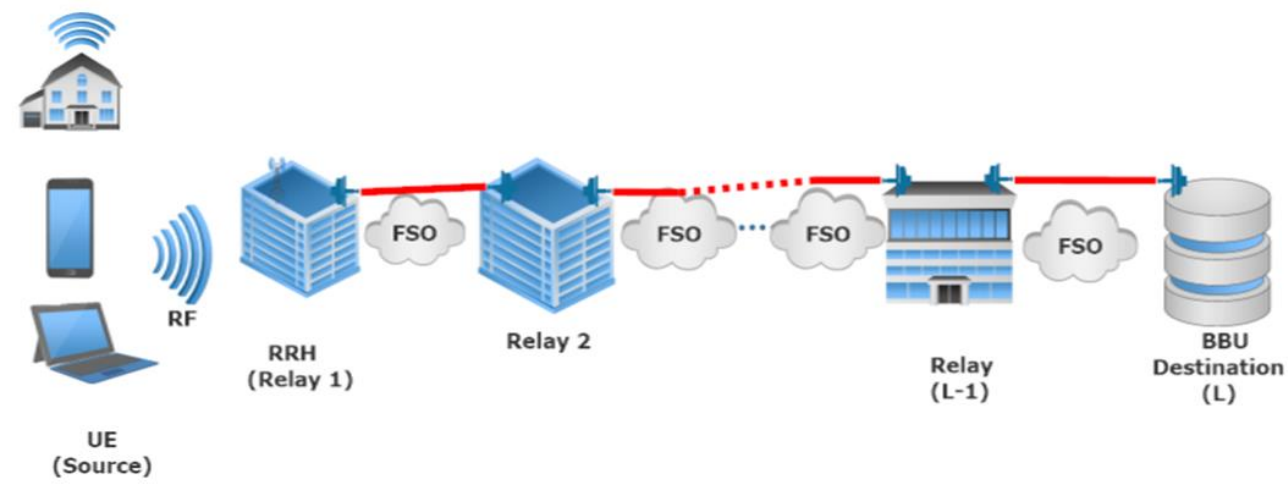

Fig. 1 Mixed RF and multihop FSO system with coherent detection

The signal from the source is transmitted to the first relay node, Relay 1 , through an RF link. The received signal from Source to Relay 1 is represented as Y1 and is given in Eq. (1) as,

$$
\mathrm{Y}_{1}=\mathrm{h}_{R_{-\{1\}}} \mathrm{X}_{1}+\mathrm{n}_{R_{-\{1\}}}
$$

where, $\mathrm{h}_{R_{-\{1\}}}$ denotes the channel gain of Generalized Nagakami-m distribution, $\mathrm{X}_{1}$ is the signal transmitted from Source to Relay 1. $\mathrm{n}_{R_{-\{1\}}}$ is the additive white Gaussian noise added in the RF channel.

From the first relay Relay 1 to the destination, the method adopted for detection is coherent. The coherent OFDM-FSO system is adapted from relay 'i' to a destination where $i=2 \ldots \mathrm{L}$ nodes. The signal transmitted from Relay 1 to Destination is $\mathrm{X}_{\mathrm{i}}$ and the received signal at the destination is denoted as $\mathrm{Y}_{\mathrm{i}}$. 


$$
\mathrm{Y}_{\mathrm{i}}=\mathrm{h}_{R_{-\{i\}}} \varepsilon_{i} \mathrm{X}_{\mathrm{i}}+\mathrm{n}_{R_{-\{i\}}}
$$

where, $\varepsilon_{i}$ is the $\mathrm{i}^{\text {th }}$ receiver sensitivity.

\subsection{Channel Model}

\subsubsection{RF link}

The RF link is established between Source and Relay 1. The generalized Nakagami m fading channel is chosen as the RF link. The instantaneous SNR PDF of the generalized Nakagami-m fading function is given as (E. Zedini et al. 2015)

$$
f \gamma_{\mathrm{RF}}\left(\gamma_{\mathrm{RF}}\right)=\frac{\mathrm{p}}{\Gamma(\mathrm{m})}\left(\frac{\mathrm{d}}{\overline{\gamma_{\mathrm{RF}}}}\right)^{\mathrm{mp}} \gamma_{\mathrm{RF}}^{\mathrm{mp}-1} \mathrm{e}^{\left(\frac{-\mathrm{d}}{\overline{\gamma_{\mathrm{RF}}}}\right)^{\mathrm{p}}} \gamma_{\mathrm{RF}}^{\mathrm{p}},
$$

Where $m(m>=1 / 2)$ and $p(p>0)$ are the fading figures and shaping parameters, respectively, $\overline{\gamma_{R F}}$ is the average SNR, and $d=\Gamma(m+1 / p) / \Gamma(m)$. For the special cases, Generalized Nakagami m fading distribution tends to Nakagami-m, Rayleigh, Exponential, Gamma and Weibull when $(p=1),(m=1, p=1),(m=1, p=1 / 2)$, $(\mathrm{p}=1 / 2)$ and $(\mathrm{m}=1)$ respectively. The PDF of Generalized Gamma distribution in terms of Meijer's G function is given as

$$
f \gamma_{\mathrm{RF}}\left(\gamma_{\mathrm{RF}}\right)=\frac{\mathrm{p}}{\Gamma(\mathrm{m}) \gamma} \mathrm{G}_{0,1}^{1,0}\left[\left.\left(\frac{\mathrm{d}}{\overline{\overline{\gamma_{\mathrm{RF}}}}}\right)^{\mathrm{p}} \gamma_{\mathrm{RF}}^{\mathrm{p}}\right|_{\mathrm{m}} ^{-}\right] .
$$

The CDF of the Generalized Gamma distribution is represented as (E. Zedini et al. 2015)

$$
\mathrm{F} \gamma_{\mathrm{RF}}\left(\gamma_{\mathrm{RF}}\right)=1-\frac{1}{\Gamma(\mathrm{m})} \mathrm{G}_{1,2}^{2,0}\left[\left(\frac{\mathrm{d}}{\overline{\gamma_{\mathrm{RF}}}}\right)^{\mathrm{p}} \gamma_{\mathrm{RF}}^{\mathrm{p}} \mid \begin{array}{c}
1 \\
\mathrm{~m}, 0
\end{array}\right] .
$$

\subsubsection{FSO link}

The FSO link is assumed Gamma-Gamma distributed. The combined channel state of Gamma Gamma distribution includes three factors, atmospheric loss $\left(\mathrm{h}_{\mathrm{li}}\right)$, pointing error $\left(\mathrm{h}_{\mathrm{pi}}\right)$, and atmospheric turbulence $\left(\mathrm{h}_{\mathrm{ai}}\right)$ (Wang et al. 2016). The combined Channel state factor $\mathrm{h}=\mathrm{h}_{\mathrm{li}} \mathrm{h}_{\mathrm{ph}} \mathrm{h}_{\mathrm{ai}}$. Atmospheric loss is represented as

$$
\mathrm{h}_{\mathrm{li}}(\mathrm{z})=\mathrm{e}^{-(\sigma \mathrm{z})}
$$


where, $\mathrm{h}_{\mathrm{li}}(\mathrm{z})$ is the loss due to attenuation when the signal propagates in a path of length $\mathrm{z}$ Kms, and $\sigma$ is the attenuation coefficient. This loss is due to suspended particles present in the atmosphere, which affects the visibility of the optical light.

Pointing error is caused due to the misalignment of the transmitter and receiver. This misalignment occurs due to the vibration between the transceivers, wind speed change, and temperature change in the atmosphere (Anbarasi. K et al., 2017).In multihop systems, the alignment between the source, relays, and destination is affected mainly by jitter errors, which must be considered when modeling the channel. Pointing errors depends on jitter error and the beamwidth.

The pointing error with a radial displacement of the beam following Gaussian distribution is represented in (I. S. Ansari et al., 2013).

$$
\mathrm{h}_{\mathrm{pi}}(\delta)=\mathrm{A}_{0} \mathrm{e}^{\left(-\frac{2 \delta^{2}}{\mathrm{w}_{\mathrm{zeq}}^{2}}\right)}
$$

Where, $\mathrm{w}_{\mathrm{zeq}}^{2}=\mathrm{w}_{\mathrm{z}}^{2} \sqrt{\pi} \operatorname{erf}(\mathrm{v}) \mid 2 \mathrm{vexp}\left(-\mathrm{v}^{2}\right)$ is the equivalent beam width, $\mathrm{v}=\sqrt{\pi} \mathrm{r} / \sqrt{2} \mathrm{w}_{\mathrm{z}} \mathrm{R}$ is the aperture radius of the receiver. $\mathrm{w}_{\mathrm{z}}$ is the beam width at link length of $\mathrm{z}, \mathrm{w}_{\mathrm{z}} / \mathrm{r}$ is the normalized beam width, $\mathrm{A}_{0}=$ $[\operatorname{erf}(\mathrm{v})]^{2}$ is the power received at $\delta=0$. The pdf of $\mathrm{h}_{\mathrm{pi}}$ is written as

$$
\mathrm{f}_{\mathrm{h}_{\mathrm{pi}}}\left(\mathrm{h}_{\mathrm{pi}}\right)=\frac{\gamma_{\mathrm{i}}^{2}}{\mathrm{~A}_{0}^{\gamma_{\mathrm{i}}^{2}}} \mathrm{~h}_{\mathrm{pi}}^{\gamma_{\mathrm{i}}^{2}-1}, 0 \leq \mathrm{h}_{\mathrm{pi}} \leq \mathrm{A}_{0}
$$

where, $\gamma_{\mathrm{i}}=\mathrm{w}_{\mathrm{zeq}} / 2 \delta_{\mathrm{s}}, \delta_{\mathrm{s}}$ is the jitter and $\delta_{\mathrm{s}} / \mathrm{r}$ is the normalized jitter error.

Gamma-Gamma distribution is modeled for atmospheric turbulence conditions like weak, moderate, and strong. The pdf of Gamma Gamma distribution has two effective optical wave intensities, i.e., small scale eddies $(\alpha)$ and large scale eddies $(\beta)$ of the scattering process. It is given as

$$
\mathrm{f}_{\mathrm{h}_{\mathrm{ai}}}\left(\mathrm{h}_{\mathrm{ai}}\right)=\frac{2(\alpha \beta)^{(\alpha+\beta) / 2}}{\Gamma(\alpha) \Gamma(\beta)} \mathrm{h}_{\mathrm{ai}}^{(\alpha+\beta-2) / 2} \mathrm{~K}_{\alpha-\beta}\left(2 \sqrt{\alpha \beta \mathrm{h}_{\mathrm{ai}}}\right), \mathrm{h}_{\mathrm{ai}}>0
$$

Where $h_{a i}$ is the scintillation intensity of the signal, $K_{n}($.$) denotes the modified Bessel function of the second$ kind of order n. $\Gamma($.$) is the Gamma function. The equations of \alpha$ and $\beta$ are 


$$
\begin{gathered}
\alpha=\exp \left[\left(\frac{0.49 \sigma_{\mathrm{i}}^{2}}{\left(1+1.11 \sigma_{\mathrm{i}}^{12 / 5}\right)^{7 / 6}}\right)-1\right]^{-1} \\
\beta=\exp \left[\left(\frac{0.51 \sigma_{\mathrm{i}}^{2}}{\left(1+0.69 \sigma_{\mathrm{i}}^{12 / 5}\right)^{5 / 6}}\right)-1\right]^{-1}
\end{gathered}
$$

Where $\sigma_{\mathrm{i}}^{2}$ is the Rytov variance given as $\sigma_{\mathrm{i}}^{2}=1.23 \mathrm{C}_{\mathrm{n}}^{2} \mathrm{k}^{7 / 6} \mathrm{~L}^{11 / 6} . \mathrm{k}=2 \pi / \lambda$ is the wavenumber; $\mathrm{L}$ is the link length and $\mathrm{C}_{\mathrm{n}}^{2}$ is the refractive index structure coefficient.

The combined PDF, which includes path loss, atmospheric turbulence, and pointing errors, is given as

$$
\begin{aligned}
& f_{h_{i}}\left(h_{i}\right)=\int f_{h_{i} \mid h_{a i}}\left(h_{i} / h_{a i}\right) f_{h_{a i}}\left(h_{a i}\right) d h_{a i} \\
& \mathrm{f}_{\mathrm{h}_{\mathrm{i} \mid \mathrm{h}_{\mathrm{ai}}}}\left(\mathrm{h}_{\mathrm{i}} / \mathrm{h}_{\mathrm{ai}}\right)=\frac{1}{\mathrm{~h}_{\mathrm{li}} \mathrm{h}_{\mathrm{ai}}} \mathrm{f}\left(\frac{\mathrm{h}_{\mathrm{i}}}{\mathrm{h}_{\mathrm{li}} \mathrm{h}_{\mathrm{ai}}}\right)=\frac{\gamma_{\mathrm{i}}^{2}}{\mathrm{~A}_{0} \gamma_{\mathrm{i}}^{2} \mathrm{~h}_{\mathrm{li}} \mathrm{h}_{\mathrm{ai}}}\left(\frac{\mathrm{h}_{\mathrm{i}}}{\mathrm{h}_{\mathrm{li}} \mathrm{h}_{\mathrm{ai}}}\right)^{\gamma_{\mathrm{i}}^{2}-1} 0 \leq \mathrm{h}_{\mathrm{i}} \leq \mathrm{A}_{0} \mathrm{~h}_{\mathrm{li}} \mathrm{h}_{\mathrm{ai}}
\end{aligned}
$$

By substituting Eqs. (7) and (9) into Eq.(10), we get

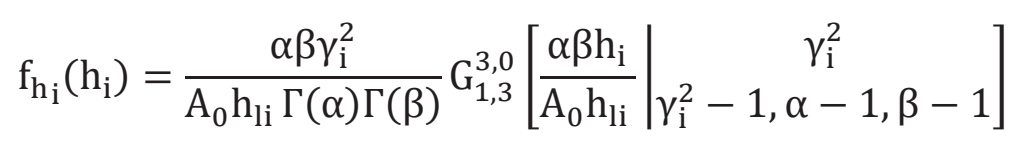

\section{Error-Correcting Codes}

\subsection{Bose-Chaudhri-Hocquenghem (BCH)}

$\mathrm{BCH}$ code is binary cyclic code in which the $\mathrm{n}-\mathrm{k}$ redundant bits are added to the $\mathrm{k}$ information bits to form a codeword of length $\mathrm{n}$ (Lin 2001; Moon 2020). A generator polynomial $\mathrm{g}(\mathrm{x})$ is used to determine the characteristics of a codeword. Multiples of generator polynomial form codewords. The information bits are represented using polynomial $\mathrm{m}(\mathrm{x})$, a codeword is formed by dividing $\mathrm{m}(\mathrm{x})$ by $\mathrm{g}(\mathrm{x})$, and the remainder is represented as check bits $r(x) . c(x)$ is the encoded data which is the summation of $m(x)$ and $r(x)$. BCH codes can correct up to $t$ independent error bits for $\mathrm{m} \geq 3$. Possible $\mathrm{BCH}$ codes when $\mathrm{m} \geq 3$ and $\mathrm{t}<2^{\mathrm{m}}-1$ are

Block length $\mathrm{n}=2^{\mathrm{m}}-1$ 
Check bits $n-k \leq m t$

Minimum distance $\mathrm{d} \geq 2 \mathrm{t}+1$ (Hamming distance)

Decoding of BCH codes follows three steps.

- From the received codeword, compute the syndrome.

- From the set of equations derived from the syndrome, find the error location polynomial.

- Identify the error bits and correct the error bits using the error location polynomial.

$\mathrm{BCH}$ codes are simple to encode and decode. Berklekamp Massey algorithm is used to decode the $\mathrm{BCH}$ coded bits.

\subsection{Reed-Solomon codes ( RS)}

Reed-Solomon codes are nonbinary cyclic codes, which belong to the BCH codes family (Lin 2001; Moon 2020). It can correct burst errors since it corrects symbol errors; even if many bits in a symbol are erroneous, it is considered as one symbol error and corrected. ' $n$ ' is the total coded symbol consisting of ' $\mathrm{m}$ ' bits per symbol, $\mathrm{k}$ is the data symbols to be encoded and $\mathrm{n}-\mathrm{k}$ is the parity symbols and is equal to $2 \mathrm{t}$, where $t$ is the capability of correcting symbol error of the code.

Possible RS codes are

$$
\begin{aligned}
& \text { Block length } \mathrm{n}=\mathrm{m}\left(2^{\mathrm{m}}-1\right) \text { bits } \\
& \text { Parity check bits } \mathrm{n}-\mathrm{k}=\mathrm{m} * 2 \mathrm{t} \\
& \text { Minimum distance } \mathrm{d}=2 \mathrm{t}+1
\end{aligned}
$$

The RS code decoding process is more complex because of the found error weight determination, i.e., the pattern of the error in the symbol has to be determined.

Reed Solomon code decoding process involves the following steps.

- Compute the syndrome from the received codeword.

- Determine the error location polynomial from the equations derived from the syndrome. 
- Determine the weight of the error from the error location polynomial.

- Correct the error symbol using the weight of the error and error location polynomial.

\section{Average Symbol Error Rate}

The influence of the atmospheric condition on the system is analyzed using a symbol error rate. As the number of relays increases, the system performance degrades. The symbol error rate of the system is derived for the M-QAM OFDM modulation technique, and the ASER is evaluated with RS and BCH codes, and the performance of un-coded and RS and BCH coded system is compared.

\subsection{RF link}

The RF link is defined by Generalized Gamma distribution. The system symbol error rate of the M-QAMOFDM system can be written as (Wang et al. 2015).

$$
\mathrm{P}_{\mathrm{QAM}-\mathrm{OFDM}}=4 \mathrm{Q}\left(\sqrt{\frac{\mathrm{h}_{\mathrm{i}}{ }^{2} 3 \mathrm{~TB}}{\mathrm{~N}(\mathrm{M}-1)} \bar{\gamma}}\right)
$$

Where $\bar{\gamma}=\frac{2 p_{\mathrm{s}}}{\mathrm{q} \rho}$, ps is the signal power, $\mathrm{q}$ is the electric charge, and $\rho$ is the detector efficiency. OFDM specifications are the number of subcarriers $(\mathrm{N})$, bandwidth $(\mathrm{B})$, and symbol period $(\mathrm{T})$. $\mathrm{M}$ is the constellation mapping coefficient of QAM.

The average symbol error probability in terms of PDF is given as

$$
P_{S E R, i}=\int_{-\infty}^{\infty} P_{e / h_{i}} f_{h_{i}}\left(h_{i}\right) d h_{i}
$$

In terms of CDF, $\mathrm{P}_{\mathrm{SER}, \mathrm{i}}=-\int_{0}^{\infty} \frac{\mathrm{dP}_{\mathrm{e}} / \mathrm{h}_{\mathrm{i}}}{\mathrm{dh}_{\mathrm{i}}} \mathrm{F}_{\mathrm{h}_{\mathrm{i}}}\left(\mathrm{h}_{\mathrm{i}}\right) \mathrm{dh}_{\mathrm{i}}$

By differentiating Eq. (13), we get

$$
\frac{\mathrm{dP}_{\mathrm{QAM}-\text { OFDM }}}{\mathrm{dh}_{\mathrm{i}}}=-\sqrt{\frac{6 \mathrm{~TB} \bar{\gamma}}{\pi \mathrm{N}(\mathrm{M}-1)}} \mathrm{e}^{-\left(\frac{\mathrm{h}_{\mathrm{i}}^{2} \text { 3TB }}{2 \mathrm{~N}(\mathrm{M}-1)} \bar{\gamma}\right)}
$$

After simplification, the average symbol error rate of the RF link with the 16-QAM OFDM system is obtained as in Eq. (17). The proof is available in Appendix A. 


$$
\mathrm{P}_{\mathrm{SER}, 1}^{\mathrm{RF}}=\frac{2}{\sqrt{\pi}} \sum_{\mathrm{j}=1}^{\mathrm{m}_{\mathrm{j}}} \mathrm{H}_{\mathrm{j}}\left\{1-\left\{\left(\frac{1}{\Gamma(\mathrm{m})} \mathrm{G}_{1,2}^{2,0}\left[(\mathrm{~d})^{\mathrm{p}}\left(\frac{\mathrm{y}_{\mathrm{j}}}{\mu \overline{\overline{\mathrm{RF}}_{\mathrm{R}}}}\right)^{\frac{\mathrm{p}}{4}} \mid \begin{array}{c}
1 \\
\mathrm{~m}, 0
\end{array}\right]\right)\right\}\right\}
$$

\subsection{FSO link}

The system symbol error rate of the 16-QAM-OFDM system is given in Eq. (13)

From Eq. (15), $\mathrm{P}_{\mathrm{SER}, \mathrm{i}}=-\int_{0}^{\infty} \frac{\mathrm{dP}_{\mathrm{QAM}-\mathrm{OFDM}}}{\mathrm{dh}_{\mathrm{i}}} \mathrm{F}_{\mathrm{h}_{\mathrm{i}}}\left(\mathrm{h}_{\mathrm{i}}\right) \mathrm{dh}_{\mathrm{i}}$

The CDF of Gamma-Gamma distribution is given as

$$
\mathrm{F}_{\mathrm{h}_{\mathrm{i}}}\left(\mathrm{h}_{\mathrm{i}}\right)=\frac{\gamma_{\mathrm{i}}^{2}}{\Gamma(\alpha) \Gamma(\beta)} \mathrm{G}_{2,4}^{3,1}\left[\frac{\alpha \beta \mathrm{h}_{\mathrm{i}}}{\mathrm{A}_{0} \mathrm{~h}_{\mathrm{li}}} \mid \begin{array}{c}
1, \gamma_{\mathrm{i}}^{2}+1 \\
\gamma_{\mathrm{i}}^{2}, \alpha, \beta, 0
\end{array}\right]
$$

Substituting Eqs. (21) and (16) in Eq. (15), with simple mathematical calculations, the ASER of FSO link with M-QAM coherent OFDM technique can be obtained as in Eq. (22). The proof is available in Appendix B.

$$
\mathrm{P}_{\mathrm{SER}, \mathrm{i}}^{\mathrm{FSO}}=\frac{2 \gamma_{\mathrm{i}}^{2}}{\sqrt{\pi} \Gamma(\alpha) \Gamma(\beta)} \sum_{j=1}^{m_{j}} H_{j} G_{2,4}^{3,1}\left[\frac{\alpha \beta}{\mathrm{A}_{0} \mathrm{~h}_{\mathrm{li}}}\left(\frac{\mathrm{y}_{\mathrm{j}}}{\mu \overline{\gamma_{\mathrm{FSO}}}}\right)^{1 / 2} \mid \begin{array}{l}
1, \gamma_{\mathrm{i}}^{2}+1 \\
\gamma_{\mathrm{i}}^{2}, \alpha, \beta, 0
\end{array}\right]
$$

$\mathrm{P}_{\mathrm{SER}, \mathrm{i}}$, denotes the symbol error rate equation of the $\mathrm{i}^{\text {th }}$ hop. For multihop, the average symbol error rate of $\mathrm{L}$ number of hops is given as (Wang et al. 2016)

$$
\mathrm{SER}_{\text {Total }}^{\mathrm{FSO}}=\sum_{\mathrm{i}=2}^{\mathrm{L}} \mathrm{P}_{\mathrm{SER}, \mathrm{i}}^{\mathrm{FSO}} \prod_{\mathrm{k}=\mathrm{i}+1}^{\mathrm{L}}\left(1-2 \mathrm{P}_{\mathrm{SER}, \mathrm{k}}^{\mathrm{FSO}}\right)
$$

By substituting Eq. (22) in Eq. (23), the total average system symbol error rate of multihop coherent M-QAM OFDM-FSO link is obtained as

$$
\begin{aligned}
\operatorname{SER}_{\text {Total }}^{\text {FSO }}= & \sum_{\mathrm{i}=2}^{\mathrm{L}}\left(\frac{2 \gamma_{\mathrm{i}}^{2}}{\sqrt{\pi} \Gamma(\alpha) \Gamma(\beta)} \sum_{j=1}^{\mathrm{m}_{\mathrm{j}}} \mathrm{H}_{\mathrm{j}} \mathrm{G}_{2,4}^{3,1}\left[\frac{\alpha \beta}{\mathrm{A}_{0} \mathrm{~h}_{\mathrm{li}}}\left(\frac{\mathrm{y}_{\mathrm{j}}}{\mu \overline{\gamma_{\mathrm{FSO}}}}\right)^{1 / 2} \mid \begin{array}{l}
1, \gamma_{\mathrm{i}}^{2}+1 \\
\gamma_{\mathrm{i}}^{2}, \alpha, \beta, 0
\end{array}\right] \prod_{\mathrm{k}=\mathrm{i}+1}^{\mathrm{L}}(1\right. \\
& \left.\left.-\frac{4 \gamma_{\mathrm{i}}^{2}}{\sqrt{\pi} \Gamma(\alpha) \Gamma(\beta)} \sum_{\mathrm{j}=1}^{\mathrm{m}_{\mathrm{j}}} \mathrm{H}_{\mathrm{j}} \mathrm{G}_{2,4}^{3,1}\left[\frac{\alpha \beta}{\mathrm{A}_{0} \mathrm{~h}_{\mathrm{li}}}\left(\frac{\mathrm{y}_{\mathrm{j}}}{\mu \overline{\gamma_{\mathrm{FSO}}}}\right)^{1 / 2} \mid \begin{array}{l}
1, \gamma_{\mathrm{i}}^{2}+1 \\
\gamma_{\mathrm{i}}^{2}, \alpha, \beta, 0
\end{array}\right]\right)\right)
\end{aligned}
$$




\subsection{Mixed RF and multihop FSO system}

In (Yi, Xiang, et al. 2019), Eq. (29) gives the ASER of mixed RF and multihop FSO system

$$
\mathrm{SER}_{\mathrm{T}}^{\text {sys }}=\frac{1}{2}-\left(\frac{1}{2}-\mathrm{P}_{\mathrm{SER}, 1}^{\mathrm{RF}}\right)\left(1-2 \mathrm{SER}_{\mathrm{Total}}^{\mathrm{FSO}}\right)
$$

ASER of mixed RF and multihop coherent FSO with 16-QAM OFDM system is obtained from Eqs. (17) and (24) as

$$
\begin{aligned}
\operatorname{SER}_{\mathrm{T}}^{\text {sys }}=\frac{1}{2}- & \left(\frac{1}{2}-\frac{2}{\sqrt{\pi}} \sum_{\mathrm{j}=1}^{\mathrm{m}_{\mathrm{j}}} \mathrm{H}_{\mathrm{j}}\left\{1-\left\{\left(\frac{1}{\Gamma(\mathrm{m})} \mathrm{G}_{1,2}^{2,0}\left[(\mathrm{~d})^{\mathrm{p}}\left(\frac{\mathrm{y}_{\mathrm{j}}}{\mu \overline{\gamma_{\mathrm{RF}}}}\right)^{\frac{\mathrm{p}}{4}} \mid \begin{array}{c}
1 \\
\mathrm{~m}, 0
\end{array}\right]\right)\right\}\right\}\right)(1 \\
& -2 \sum_{\mathrm{i}=2}^{\mathrm{L}}\left(\frac{2 \gamma_{\mathrm{i}}^{2}}{\sqrt{\pi} \Gamma(\alpha) \Gamma(\beta)} \sum_{\mathrm{j}=1}^{\mathrm{m}_{\mathrm{j}}} \mathrm{H}_{\mathrm{j}} \mathrm{G}_{2,4}^{3,1}\left[\frac{\alpha \beta}{\mathrm{A}_{0} \mathrm{~h}_{\mathrm{li}}}\left(\frac{\mathrm{y}_{\mathrm{j}}}{\mu \overline{\gamma_{\mathrm{FSO}}}}\right)^{1 / 2} \mid \begin{array}{c}
1, \gamma_{\mathrm{i}}^{2}+1 \\
\gamma_{\mathrm{i}}^{2}, \alpha, \beta, 0
\end{array}\right] \prod_{\mathrm{k}=\mathrm{i}+1}^{\mathrm{L}}(1\right. \\
& \left.\left.\left.-\frac{4 \gamma_{\mathrm{i}}^{2}}{\sqrt{\pi} \Gamma(\alpha) \Gamma(\beta)} \sum_{\mathrm{j}=1}^{\mathrm{m}_{\mathrm{j}}} \mathrm{H}_{\mathrm{j}} \mathrm{G}_{2,4}^{3,1}\left[\frac{\alpha \beta}{\mathrm{A}_{0} \mathrm{~h}_{\mathrm{li}}}\left(\frac{\mathrm{y}_{\mathrm{j}}}{\mu \overline{\gamma_{\mathrm{FSO}}}}\right)^{1 / 2} \mid \begin{array}{c}
1, \gamma_{\mathrm{i}}^{2}+1 \\
\gamma_{\mathrm{i}}^{2}, \alpha, \beta, 0
\end{array}\right]\right)\right)\right)
\end{aligned}
$$

\subsection{Coded SER analysis}

Mixed RF and multihop 16-QAM modulated OFDM system is coded with RS and BCH codes with the code rate of 0.733 . When a non-identical codeword from the transmitted codeword is received, an error occurs. The probability of decoded error for ( $\mathrm{n}, \mathrm{k}, \mathrm{t}) \mathrm{RS}$ and $\mathrm{BCH}$ codes with $\mathrm{t}$ error-correcting capability is given by (Ramavath Prasad Naik 2020; Ramavath Prasad Naik 2020)

$$
P_{B C H} \leq \sum_{i=t+1}^{n}\left(\begin{array}{c}
n \\
i
\end{array}\right) p^{i}(1-p)^{n-i}
$$

where $\mathrm{p}$ is the symbol error probability. Here $\mathrm{p}$ is the $\mathrm{SER}_{\mathrm{T}}^{\text {sys }}$ from equation (30), substituting it in equation (31) gives $\mathrm{P}_{\mathrm{BCH}}$ of the system. 


$$
P_{R S} \leq \sum_{i=t+1}^{n}\left(\begin{array}{l}
n \\
i
\end{array}\right) p_{S}^{i}\left(1-p_{S}\right)^{n-i}
$$

where $p_{s}=1-\left(1-p_{b}\right)^{m}, p_{b}$ represents the system error probability. Substituting equation (30) in the place of $p_{b}$ gives $P_{R S}$.

\section{Outage Probability}

The outage probability $\mathrm{P}_{\text {out }}$ of the multihop FSO system is given as

$$
\begin{aligned}
& \mathrm{P}_{\text {out }}=\mathrm{P}_{\mathrm{ri}}\left[\min \left(\mu_{\mathrm{ri}}\right) \leq \mu_{\mathrm{thr}}\right] \\
& =1-\prod_{\mathrm{i}=1}^{\mathrm{L}}\left(1-\mathrm{P}_{\text {out }_{\mathrm{FSO}}}\left(\mu_{\mathrm{thr}}\right)\right)
\end{aligned}
$$

The $\mathrm{P}_{\text {out }}$ is given as

$$
\mathrm{P}_{\text {out }}=1-\left(\left(1-\mathrm{P}_{\text {out }_{\mathrm{RF}}}\right) \times \prod_{\mathrm{i}=1}^{\mathrm{L}}\left(1-\mathrm{P}_{\text {out }_{\mathrm{FSO}}}\left(\mu_{\mathrm{thr}}\right)\right)\right)
$$

For a multihop coherent OFDM system, the outage probability is given as (Wang et al. 2016)

$$
\mathrm{P}_{\text {out_FSO }}=1-\prod_{\mathrm{i}=1}^{\mathrm{L}}\left(1-\frac{\alpha \beta \gamma_{\mathrm{i}}^{2} \mathrm{x} \sqrt{\mathrm{N} / 2 \mathrm{~T}}}{\mathrm{~A}_{0} \mathrm{~h}_{\mathrm{li}} \Gamma(\alpha) \Gamma(\beta)} \mathrm{G}_{2,4}^{3,1}\left[\frac{\alpha \beta \mathrm{x} \sqrt{\mathrm{N} / \mathrm{T}}}{\mathrm{A}_{0} \mathrm{~h}_{\mathrm{li}}} \mid \begin{array}{c}
1, \gamma_{\mathrm{i}}^{2}+1 \\
\gamma_{\mathrm{i}}^{2}, \alpha, \beta, 0
\end{array}\right]\right)
$$

The outage probability of mixed RF and multihop coherent FSO-OFDM systems is obtained by substituting Eqs. (35) and (5) in Eq. (34) and we get,

$$
\begin{aligned}
\mathrm{P}_{\text {out }}=1- & \left\{\left(\frac{1}{\Gamma(\mathrm{m})} \mathrm{G}_{1,2}^{2,0}\left[\left(\frac{\mathrm{d}}{\overline{\gamma_{\mathrm{RF}}}}\right)^{\mathrm{p}} \gamma_{\mathrm{RF}}^{\mathrm{p}} \mid \begin{array}{c}
1 \\
\mathrm{~m}, 0
\end{array}\right]\right)\right. \\
& \times\left(1-\prod_{\mathrm{i}=1}^{\mathrm{L}}\left(1-\frac{\alpha \beta \gamma_{\mathrm{i}}^{2} \mathrm{x} \sqrt{\mathrm{N} / 2 \mathrm{~T}}}{\mathrm{~A}_{0} \mathrm{~h}_{\mathrm{li}} \Gamma(\alpha) \Gamma(\beta)} \mathrm{G}_{2,4}^{3,1}\left[\frac{\alpha \beta \mathrm{x} \sqrt{\mathrm{N} / \mathrm{T}}}{\mathrm{A}_{0} \mathrm{~h}_{\mathrm{li}}} \mid \begin{array}{c}
1, \gamma_{\mathrm{i}}^{2}+1 \\
\gamma_{\mathrm{i}}^{2}, \alpha, \beta, 0
\end{array}\right]\right)\right\}
\end{aligned}
$$




\section{Results and discussions}

In this section, the outage probability and the error performance of mixed RF and multihop coherent OFDM-FSO systems are analyzed under the influence of atmospheric turbulence, various weather conditions, and pointing errors.

Table 1 aand $\beta$ values of turbulence conditions (Wang et al. 2016)

\begin{tabular}{lccc}
\hline S.No. & Turbulence & $\boldsymbol{\alpha}$ & $\boldsymbol{\beta}$ \\
\hline 1 & Weak & 6.05 & 4.47 \\
2 & Moderate & 4.19 & 2.26 \\
3 & Strong & 4.34 & 1.31
\end{tabular}

Table 2 Attenuation values for different weather conditions (Duan, Mengyi et al. 2018)

\begin{tabular}{lll}
\hline S.No & Weather & Attenuation \\
& condition & $\mathbf{( 3 d B / K m ) ~}$ \\
\hline 1 & Very clear air & 0.0647 \\
2 & Clear air/drizzle & 0.2208 \\
3 & Haze & 0.7360 \\
4 & Light fog & 4.2850
\end{tabular}

The error performance of the uncoded and coded mixed RF and multihop coherent OFDM-FSO system is compared. Both RF and multihop FSO systems follow M-QAM modulated OFDM system. The numerical results are validated using Monte Carlo Simulations. Numerical analysis is done for moderate and strong atmospheric turbulence conditions. The scintillation parameter values are given in Table I. For the OFDM system, the number of subcarriers is taken as $\mathrm{N}=256$. The number of hops considered in this work is two and five. For QAM modulation M is taken as 16 . BCH $(15,11)$ and $\mathrm{RS}(15,11)$ are applied to the uncoded system. 


\subsection{ASER of mixed RF and multihop coherent OFDM-FSO link}

\subsubsection{Atmospheric turbulence effect}

The effect of the atmospheric turbulence (moderate and strong) on the uncoded and coded system is analyzed under a constant path loss of 0.44 . The pointing error is considered constant with beamwidth and jitter error $\mathrm{w}_{\mathrm{z}} / \mathrm{r}=25$ and $\delta_{\mathrm{s}} / \mathrm{r}=3$ respectively. Fig. 2 shows the ASER performance for different hop numbers. The atmospheric turbulence condition is varied as moderate and strong turbulence. From Fig. 2, it is clear that an increase in the intensity of the scintillation $\left(\mathrm{C}_{\mathrm{n}}^{2}\right)$ increases the symbol error rate, increase in the average signal to noise ratio decreases the average symbol error rate. The error performance of the system is affected when the number of relays increases. When the number of hops increases from $\mathrm{L}=2$ to $\mathrm{L}=5$, the system performance degrades due to system interruption.

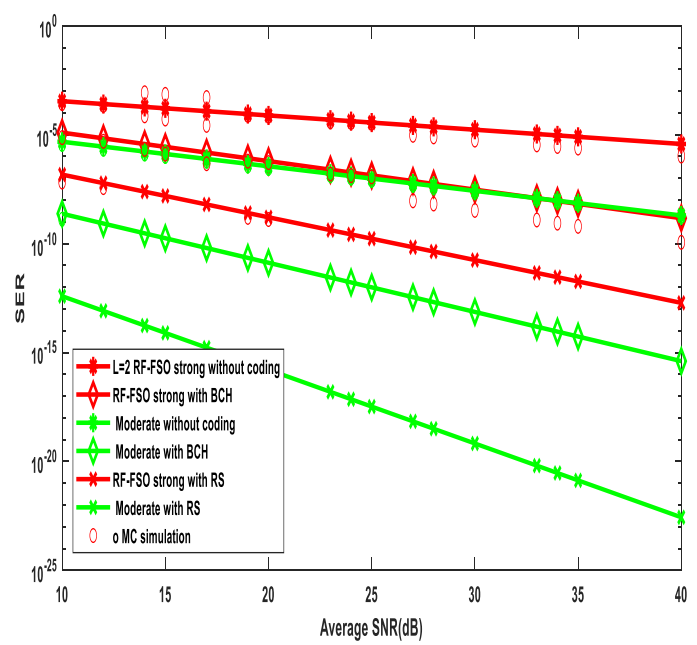

(a)

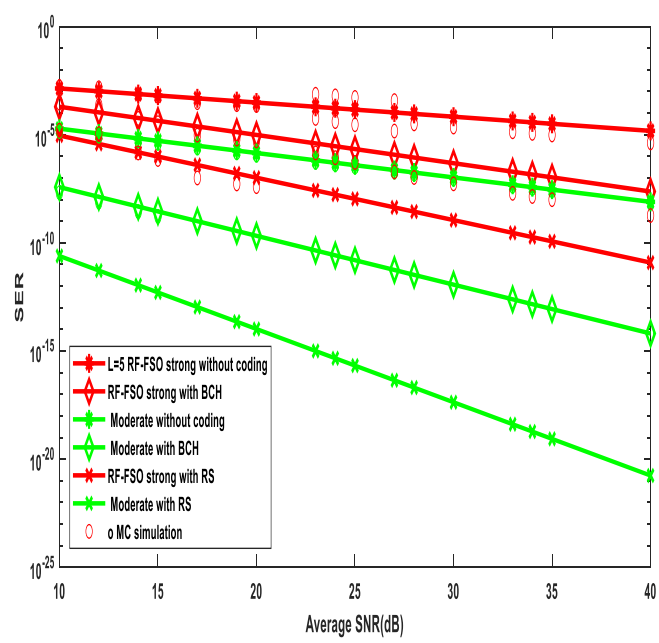

(b)

Fig. 2 Mixed RF and multihop coherent FSO system with 16-QAM OFDM with MC simulation for path loss is 0.44 , pointing error with $\mathrm{w}_{\mathrm{z}} / \mathrm{r}=25$ and $\delta_{\mathrm{s}} / \mathrm{r}=3$ for $\mathbf{a} \mathrm{L}=2$ and $\mathbf{b} \mathrm{L}=5$ hops.

Fig. 2a and Fig. 2b show that the system's performance is improved when a coding technique is used. The advantages of a coherent OFDM system enhance the performance of the system by reducing the error rate. At $15 \mathrm{~dB}$, for strong turbulence condition, uncoded, BCH coded, and RS coded system attains SER of $10^{-4}, 10^{-5}$, 
and $10^{-7}$, respectively. As the average SNR increases, system performance increases. RS codes give better SNR gain than $\mathrm{BCH}$ codes.

\subsubsection{Effect of Weather conditions}

It is known that weather conditions have an adverse effect on the FSO system performance, and fog affects the FSO link as it hinders the line of sight. ASER performance is analyzed by varying the weather conditions for strong turbulence conditions and pointing error with beam width and jitter error as wz/r=25 and $\delta \mathrm{s} / \mathrm{r}=3$.

From Fig. $3 a$ and $3 b$, it is clear that the influence of fog is high when compared with other weather conditions like clear air, drizzle, and haze. At $10 \mathrm{~dB}$ SNR, ASER is below $10^{-3}$ for all weather conditions except fog. For fog, when $\mathrm{L}=5$ hops, as the SNR increases above $30 \mathrm{~dB}$, ASER reaches $10^{-1}$, and for haze at $15 \mathrm{~dB}$ SNR, ASER of the uncoded system is $10^{-4}$, BCH coded system is $10^{-5}$, and RS coded system is $10^{-6}$. Fig. $3 \mathrm{a}$ and Fig $3 b$, as the relay increases, system interruption increases, and SER increases.

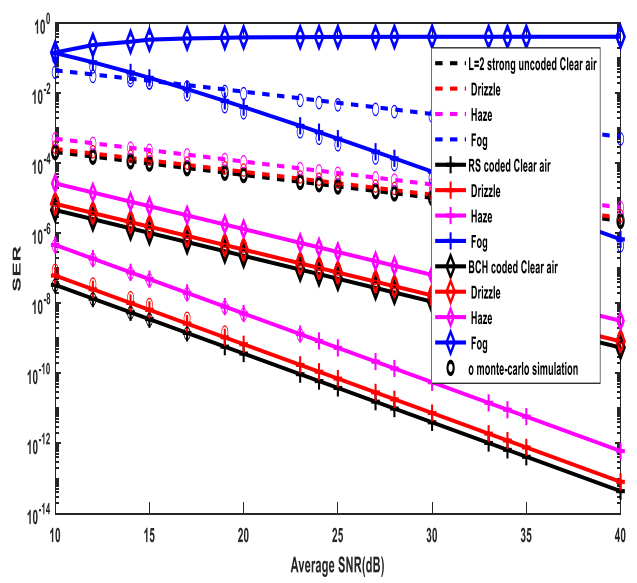

(a)

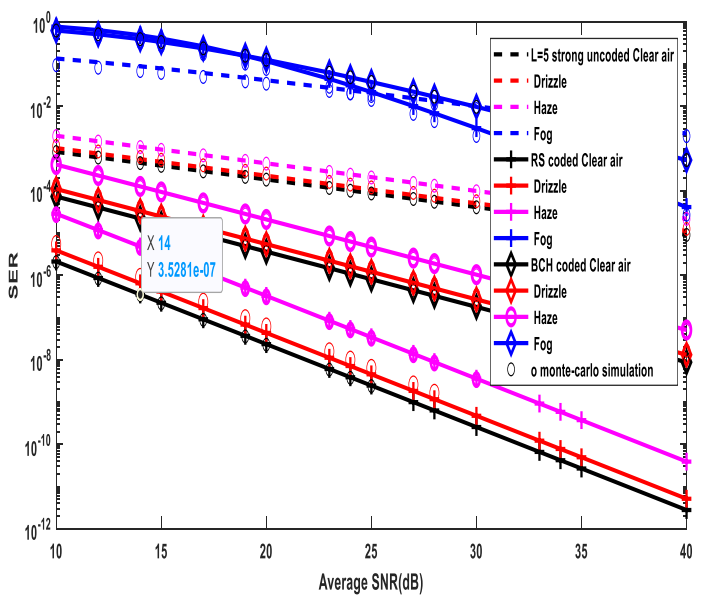

(b)

Fig. 3 Different weather conditions with Strong turbulence condition and pointing error with beam width and jitter error as $\mathrm{w}_{\mathrm{Z}} / \mathrm{r}=25$ and $\delta_{\mathrm{s}} / \mathrm{r}=3$ for $\mathbf{a} \mathrm{L}=2$ hops and $\mathbf{b} \mathrm{L}=5$ hops

The performance of the system depends on the FSO link. FSO link is less affected by clear air and drizzle, whereas system performance deteriorates for adverse weather conditions like haze and fog. Compared to the 
uncoded system, the coded system improves the system performance. In the coding system, RS codes perform better than $\mathrm{BCH}$ codes.

\subsubsection{Effect of Pointing error}

The effect of pointing error is analyzed by varying the normalized beam width and jitter error. Beamwidth $\mathrm{W}_{\mathrm{Z}} / \mathrm{r}$ is increased from 2 to 5 , and jitter error $\delta_{\mathrm{S}} / \mathrm{r}$ is increased from 2 to 5 . The analysis is performed for strong turbulence and path loss as 0.44 with average SNR of $35 \mathrm{~dB}$.

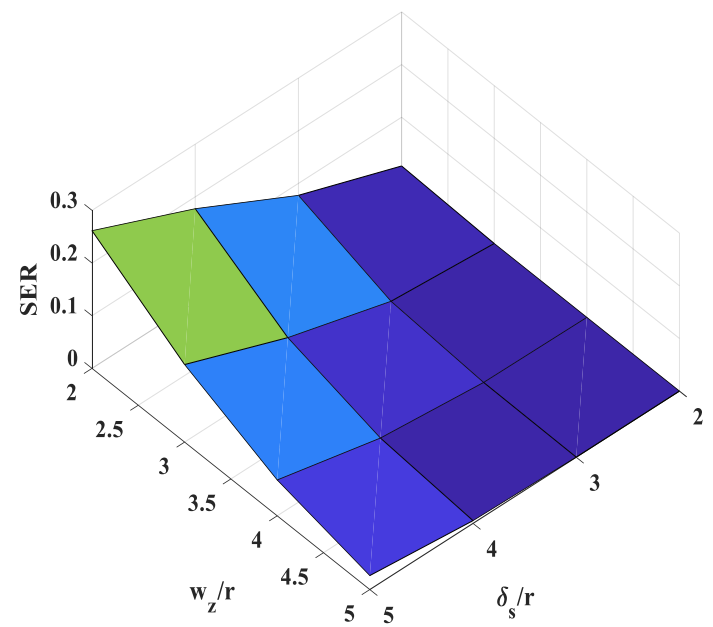

(a)

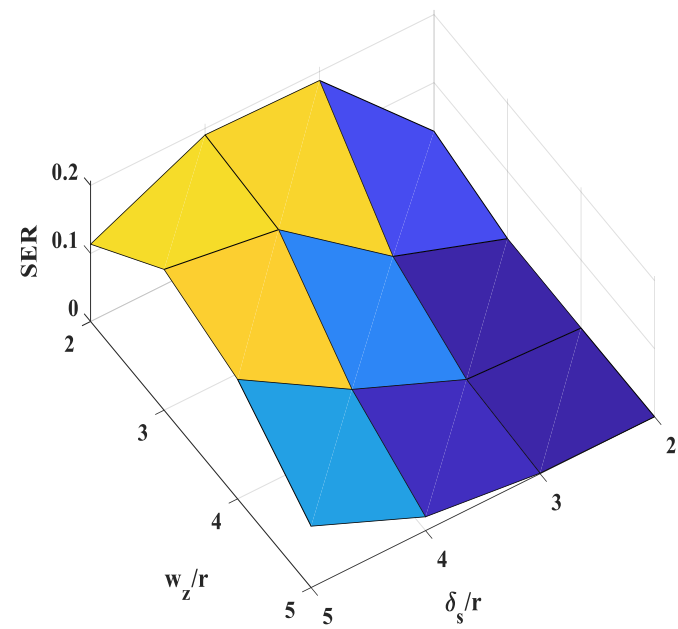

(b)

Fig. 4 Path loss as 0.44 and Strong turbulence condition $\mathbf{a} \mathrm{L}=2$ hops and $\mathbf{b} \mathrm{L}=5$ hops

From Fig. 4, it is shown that increases in beam width decrease the SER of the system, whereas an increase in jitter error increases the SER. An increase in beam width from 2 to 3 decreases the SER by 0.0072 for $L=2$ hops, and for L=5 hops, SER falls by 0.017 . When the width of the beam is increased, it leads to better SER performance than narrow beamwidth under the condition of misalignment (Wang, Ping, et al., 2015). SER performance degradation due to an increase in the number of hops can be reduced by increasing the beamwidth. An increase in jitter error from 4 to 5 for beam width 5 increases the SER by 0.0213 and 0.069 for $\mathrm{L}=2$ and five hops, respectively. The lower value of jitter does not have much impact on system performance when the strength of the jitter increases; alignment between the transmitter and receiver is worsening, which leads to degradation in the performance of the multihop system. 


\subsection{Outage Probability}

Outage Probability and the system cost increases with the increase in the number of relays. Outage probability is measured under different atmospheric turbulence conditions with fixed path loss, weather, and pointing error. The beam width and jitter error are taken as $\mathrm{w}_{\mathrm{z}} / \mathrm{r}=25$ and $\delta_{\mathrm{s}} / \mathrm{r}=3$, respectively (Wang et al. 2016). The outage performance of the single RF link and serial relaying FSO system is analyzed for the different number of hops. For the RF link, the fading figure and the shape parameter value are taken as $\mathrm{m}=2.5$ and $\mathrm{p}=1.15$, respectively (E. Zedini et al. 2016) for the generalized gamma fading channel. The outage probability of the system decreases with the decrease in the normalized threshold value. As the normalized threshold value increases, the Outage probability increases and reaches 1 for more than $-10 \mathrm{~dB}$. As the number of relays increases, the outage probability increases since the system's outage increases as the number of hops increases. From Fig. 5, for the normalized threshold value of $-15 \mathrm{~dB}$, as the number of hops rises from $\mathrm{L}=2$ to $\mathrm{L}=5$, outage probability increases by 0.005 for moderate turbulence and 0.007 for strong turbulence. Along with the increase in the hop numbers, an increase in scintillation intensity increases the system's outage. 


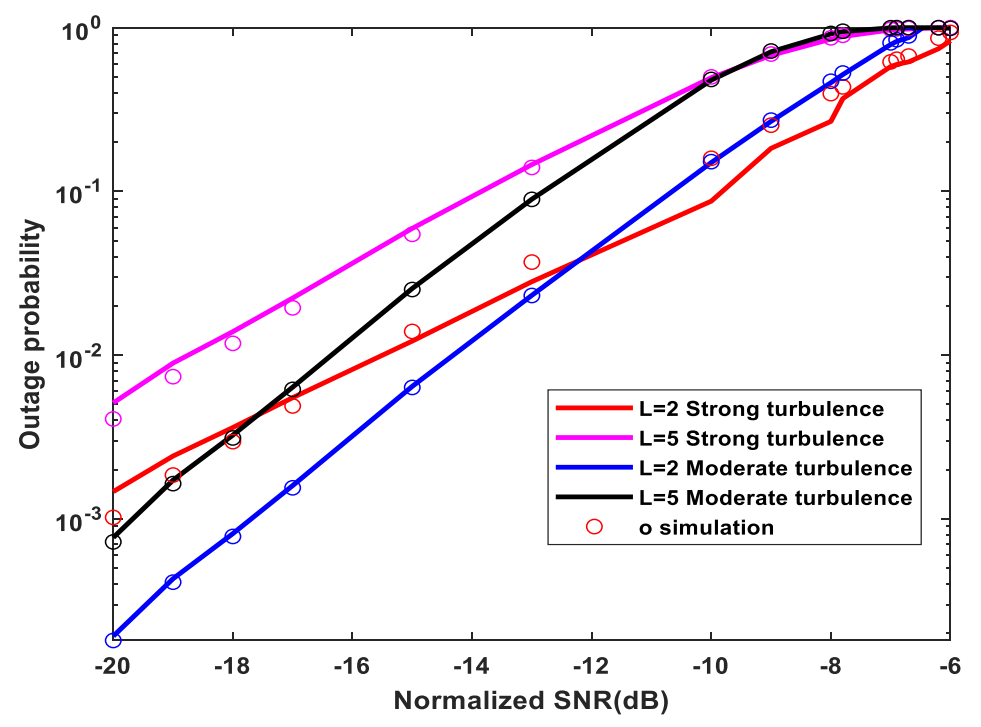

Fig. 5 OP system with MC simulation for path loss of 0.44 , pointing error with beam width and jitter error as $\mathrm{w}_{\mathrm{z}} / \mathrm{r}=25$ and $\delta_{\mathrm{s}} / \mathrm{r}=3$ for Coherent OFDM-FSO system

\section{Conclusion}

OP and ASER of the singe RF and multihop coherent OFDM-FSO system are derived for RF link with Generalized Nakagami-m fading channel and FSO with Gamma-Gamma distribution. Performance analysis is done for coherent OFDM-FSO system, which follows DF relaying scheme. The influence of different atmospheric turbulence conditions, weather conditions, and pointing errors on uncoded and coded systems is shown. The analytical results show that an increase in hop numbers in both uncoded and coded systems increases the system interruption in extending the coverage area. FSO link performance is severely affected by fog in both detection schemes. But the coded system improves the performance of the uncoded system. In a coded system, usage of RS codes improves the system's performance more efficiently than $\mathrm{BCH}$ codes.

\section{Appendix A.}

By substituting Eqs. (16) and (5) in Eq. (15), the average symbol error rate of the RF link with 16-QAM OFDM system is 


$$
\mathrm{P}_{\mathrm{SER}, 1}^{\mathrm{RF}}=2 \int_{0}^{\infty} \sqrt{\frac{6 \mathrm{~TB} \bar{\gamma}}{\pi \mathrm{N}(\mathrm{M}-1)}} \mathrm{e}^{-\left(\frac{\mathrm{h}_{\mathrm{i}}^{2} 3 \mathrm{~TB}}{2 \mathrm{~N}(\mathrm{M}-1)} \bar{\gamma}\right)} \times\left\{1-\left\{\left(\frac{1}{\Gamma(\mathrm{m})} \mathrm{G}_{1,2}^{2,0}\left[\left(\frac{\mathrm{d}}{\overline{\gamma_{\mathrm{RF}}}}\right)^{\mathrm{p}} \gamma_{\mathrm{RF}}^{\mathrm{p}} \mid \begin{array}{c}
1 \\
\mathrm{~m}, 0
\end{array}\right]\right)\right\}\right\} \mathrm{dh}_{\mathrm{i}}
$$

Where, $\gamma=\bar{\gamma} \mathrm{h}_{\mathrm{i}}^{2}$, so Eq. (A1) becomes,

$$
\mathrm{P}_{\mathrm{SER}, 1}^{\mathrm{RF}}=2 \int_{0}^{\infty} \sqrt{\frac{6 \mathrm{~TB} \bar{\gamma}}{\pi \mathrm{N}(\mathrm{M}-1)}} \mathrm{e}^{-\left(\frac{\mathrm{h}_{\mathrm{i}}^{2} 3 \mathrm{~TB}}{2 \mathrm{~N}(\mathrm{M}-1)} \bar{\gamma}\right)} \times\left\{1-\left\{\left(\frac{1}{\Gamma(\mathrm{m})} \mathrm{G}_{1,2}^{2,0}\left[\left.(\mathrm{~d})^{\mathrm{p}}\left(\mathrm{h}_{\mathrm{i}}\right)^{\frac{\mathrm{p}}{2}}\right|_{\mathrm{m}, 0} ^{1}\right]\right)\right\}\right\} \mathrm{dh}_{\mathrm{i}}
$$

Assume, $y=\frac{\mathrm{h}_{\mathrm{i}}^{2} 3 \mathrm{~TB} \bar{\gamma}}{2 \mathrm{~N}(\mathrm{M}-1)}$, then Eq. (A2) becomes

$$
\mathrm{P}_{\mathrm{SER}, 1}^{\mathrm{RF}}=\frac{2}{\sqrt{\pi}} \int_{0}^{\infty} \mathrm{y}^{-\frac{1}{2}} \mathrm{e}^{-\mathrm{y}} \times\left\{1-\left\{\left(\frac{1}{\Gamma(\mathrm{m})} \mathrm{G}_{1,2}^{2,0}\left[(\mathrm{~d})^{\mathrm{p}}\left(\frac{2 \mathrm{~N}(\mathrm{M}-1) \mathrm{y}}{3 \mathrm{~TB} \overline{\gamma_{\mathrm{RF}}}}\right)^{\frac{\mathrm{p}}{4}} \mid \begin{array}{c}
1 \\
\mathrm{~m}, 0
\end{array}\right]\right)\right\}\right\} \mathrm{dy}
$$

The above equation is of the form $\int_{0}^{\infty} x^{-\frac{1}{2}} e^{-x} f(x) d x$, the mixed RF and multihop coherent FSO-OFDM system with the affecting factors like atmospheric turbulence, path loss, and pointing error can be approximated using the Generalized Gauss-Laguerre Quadrature function

$$
\int_{0}^{\infty} x^{-\frac{1}{2}} e^{-x} f(x) d x=\sum_{j=1}^{m_{j}} H_{j} f\left(x_{j}\right)
$$

Where

$$
H_{j}=\frac{\Gamma(n+1 / 2) x_{j}}{n !(n+1)^{2}\left[L_{n-1}^{\left(-\frac{1}{2}\right)}\left(x_{i}\right)\right]^{2}}
$$

Considering, $\mu=\frac{3 \mathrm{~TB}}{2 \mathrm{~N}(\mathrm{M}-1)}$, Eq. (A3) can be written as

$$
\mathrm{P}_{\mathrm{SER}, 1}^{\mathrm{RF}}=\frac{2}{\sqrt{\pi}} \sum_{\mathrm{j}=1}^{\mathrm{m}_{\mathrm{j}}} \mathrm{H}_{\mathrm{j}}\left\{1-\left\{\left(\frac{1}{\Gamma(\mathrm{m})} \mathrm{G}_{1,2}^{2,0}\left[(\mathrm{~d})^{\mathrm{p}}\left(\frac{\mathrm{y}_{\mathrm{j}}}{\mu \overline{\gamma_{\mathrm{RF}}}}\right)^{\frac{\mathrm{p}}{4}} \mid \begin{array}{c}
1 \\
\mathrm{~m}, 0
\end{array}\right]\right)\right\}\right\}
$$

\section{Appendix B}

Substituting Eqs. (A5) and (16) in Eq. (15), we get 


$$
\mathrm{P}_{\mathrm{SER}, \mathrm{i}}^{\mathrm{FSO}}=\frac{2 \gamma_{\mathrm{i}}^{2}}{\Gamma(\alpha) \Gamma(\beta)} \int_{0}^{\infty} \sqrt{\frac{6 \mathrm{~TB} \bar{\gamma}}{\pi \mathrm{N}(\mathrm{M}-1)}} \mathrm{e}^{-\left(\frac{\mathrm{h}_{\mathrm{i}}^{2} \text { 3тв }}{2 \mathrm{~N}(\mathrm{M}-1)} \bar{\gamma}\right)} \times \mathrm{G}_{2,4}^{3,1}\left[\frac{\alpha \beta \mathrm{h}_{\mathrm{i}}}{\mathrm{A}_{0} \mathrm{~h}_{\mathrm{li}}} \mid \begin{array}{l}
1, \gamma_{\mathrm{i}}^{2}+1 \\
\gamma_{\mathrm{i}}^{2}, \alpha, \beta, 0
\end{array}\right] \mathrm{dh} \mathrm{h}_{\mathrm{i}}
$$

Assume, $y=\frac{\mathrm{h}_{\mathrm{i}}^{2} 3 \mathrm{~TB} \bar{\gamma}}{2 \mathrm{~N}(\mathrm{M}-1)}$, then Eq. (B1) becomes

$$
\mathrm{P}_{\mathrm{SER}, \mathrm{i}}^{\mathrm{FSO}}=\frac{\frac{2 \gamma_{\mathrm{i}}^{2}}{\Gamma(\alpha) \Gamma(\beta)}}{\sqrt{\pi}} \int_{0}^{\infty} \mathrm{y}^{-\frac{1}{2}} \mathrm{e}^{-\mathrm{y}} \times \mathrm{G}_{2,4}^{3,1}\left[\frac{\alpha \beta}{\mathrm{A}_{0} \mathrm{~h}_{\mathrm{li}}}\left(\frac{2 \mathrm{~N}(\mathrm{M}-1) \mathrm{y}}{3 \mathrm{~TB} \overline{\gamma_{\mathrm{FSO}}}}\right)^{1 / 2} \mid \begin{array}{l}
1, \gamma_{\mathrm{i}}^{2}+1 \\
\gamma_{\mathrm{i}}^{2}, \alpha, \beta, 0
\end{array}\right] \mathrm{dy}
$$

Using Generalized Gauss-Laguerre quadrature function and considering, $\mu=\frac{3 \mathrm{~TB}}{2 \mathrm{~N}(\mathrm{M}-1)}$, Eq. (B2) can be written as

$$
\mathrm{P}_{\mathrm{SER}, \mathrm{i}}^{\mathrm{FSO}}=\frac{2 \gamma_{\mathrm{i}}^{2}}{\sqrt{\pi} \Gamma(\alpha) \Gamma(\beta)} \sum_{j=1}^{m_{j}} \mathrm{H}_{\mathrm{j}} \mathrm{G}_{2,4}^{3,1}\left[\frac{\alpha \beta}{\mathrm{A}_{0} \mathrm{~h}_{\mathrm{li}}}\left(\frac{\mathrm{y}_{\mathrm{j}}}{\mu \overline{\gamma_{\mathrm{FSO}}}}\right)^{1 / 2} \mid \begin{array}{l}
1, \gamma_{\mathrm{i}}^{2}+1 \\
\gamma_{\mathrm{i}}^{2}, \alpha, \beta, 0
\end{array}\right]
$$

\section{References}

Iovanna, Paola, et al.: Future proof optical network infrastructure for 5G transport. Journal of Optical Communications and Networking. 8(12), B80-B92 (2016).

Zhang, Haijun, et al.: Fronthauling for 5G LTE-U ultra-dense cloud small cell networks. IEEE Wireless Communications. 23(6), 48-53 (2016).

Ranaweera, Chathurika, et al.: 5G C-RAN architecture: A comparison of multiple optical front haul networks. In: 2017 International Conference on Optical Network Design and Modeling (ONDM), IEEE (2017).

Ahmed, Khaled, and Steve Hranilovic.: C-RAN uplink optimization using mixed radio and FSO front haul. IEEE/OSA Journal of Optical Communications and Networking. 10(6), 603-612 (2018).

E. Lee, J. Park, D. Han, and G. Yoon.: Performance analysis of the asymmetric dual-hop relay transmission with mixed rf/fso links. IEEE Photonics Technology Letters. 23(21), 1642-1644 (2011).

I. S. Ansari, F. Yilmaz, and M.-S. Alouini.: Impact of pointing errors on the performance of mixed rf/fso 
dual-hop transmission systems. IEEE Wireless Communications Letters. 2(3), 351-354 (2013).

E. Zedini, I. S. Ansari, and M.-S.Alouini.: Performance analysis of mixed Nakagami-m and gamma-gamma dual-hop fso transmission systems. IEEE Photonics Journal. 7(1), 1-20 (2015).

E. Zedini, H. Soury, and M.-S.Alouini.: On the performance analysis of dual-hop mixed FSO/RF systems. IEEE Transactions on Wireless Communications. 15(5), 3679-3689 (2016).

Zedini.Emna, Imran Shafique Ansari, and Mohamed-Slim Alouini.: Unified performance analysis of mixed line of sight RF-FSO fixed gain dual-hop transmission systems. In: 2015 IEEE Wireless Communications and Networking Conference (WCNC).IEEE (2015).

Anees, S, and M. R. Bhatnagar.: Performance evaluation of decode-and-forward dual-hop asymmetric radio frequency-free space optical communication system. IET OPTOELECTRON. 9(5), 232-240 (2015).

Sharma, Nikhil, AnkurBansal, and ParulGarg.: Decode-and-forward relaying in mixed $\eta-\mu$ and gammagamma dual-hop transmission system. IET Communications. 10(14), 1769-1776 (2016).

Akella, Jayasri, Murat Yuksel, and Shivkumar Kalyanaraman.: Error analysis of multihop free-space optical communication. In: IEEE International Conference on Communications, 2005.ICC 2005. 2005. Vol. 3. IEEE (2005).

Tang, Xuan, et al.: Multihop free-space optical communications over turbulence channels with pointing errors using heterodyne detection. Journal of Lightwave Technology. 32(15), 2597-2604 (2014).

Bekkali, Abdelmoula, et al.: Transmission analysis of OFDM-based wireless services over turbulent radioon-FSO links modeled by gamma-gamma distribution. IEEE photonics journal 2(3), 510-520 (2010).

Chen, Chen, et al.: MDPSK-based non-equalization OFDM for coherent free-space optical communication. IEEE Photonics Technology Letters. 26(16), 1617-1620 (2014).

Wang, Yi, Deli Wang, and Jing Ma.: On the performance of coherent OFDM systems in free-space optical communications. IEEE photonics journal 7(4), 1-10 (2015).

Wayne, David T., et al.: Comparing the log-normal and gamma-gamma model to experimental probability density functions of aperture averaging data. In: Free-Space Laser Communications X. Vol. 7814.International Society for Optics and Photonics (2010). 
Wang, Yi, Deli Wang, and Jing Ma.: Performance analysis of multihop coherent OFDM free-space optical communication systems. Optics Communications. 376, 35-40 (2016).

Gupta, Nancy, Abhishek Dixit, and Virender Kumar Jain.: Performance analysis of BCH and repetition codes in gamma-gamma faded FSO link. In: 2019 National Conference on Communications (NCC). $\operatorname{IEEE}(2019)$.

Yu, Meng, Jing Li, and Jennifer C. Ricklin.: Efficient forward error correction coding for free-space optical communications. In: Free-Space Laser Communications IV. Vol. 5550. International Society for Optics and Photonics (2004).

Nayak, Anuj.: Performance analysis of LT codes and BCH codes in RF and FSO Wireless Sensor Networks. In: 2014 International Conference on Advances in Computing, Communications, and Informatics (ICACCI). IEEE (2014).

Anbarasi. K, C. Hemanth, and R. G. Sangeetha.: A review on channel models in free-space optical communication systems. Optics \& Laser Technology. 97, 161-171(2017).

Lin, Shu, and Daniel J. Costello.: Error control coding. Vol. 2. No. 4. Prentice hall (2001).

Moon, Todd K.: Error correction coding: mathematical methods and algorithms. John Wiley \& Sons (2020).

Yi, Xiang, et al.: Performance analysis for a mixed RF and multihop FSO communication system in 5G CRAN. Journal of Optical Communications and Networking 11(8), 452-464 (2019).

Ramavath Prasad Naik, Shripathi Acharya Udupi, and Prabu Krishnan.: Experimental demonstration and analysis of underwater wireless optical communication link: Design, $\mathrm{BCH}$ coded receiver diversity over the turbid and turbulent seawater channels. Microwave and Optical Technology Letters 62(6), 2207-2216 (2020).

Ramavath Prasad Naik, Shripathi Acharya Udupi, and Prabu Krishnan.: High-speed and reliable underwater wireless optical communication system using multiple-input multiple-output and channel coding techniques for IoT applications. Optics Communications 461, 125229 (2020).

Wang, Ping, et al.: Performance analysis for relay-aided multihop BPPM FSO communication system over 
exponentiated Weibull fading channels with pointing error impairments. IEEE Photonics Journal 7(4), 120 (2015).

Duan, Mengyi, et al.: Average bit error rate performance analysis of a low-density parity-check-coded orthogonal frequency-division multiplexing FSO system under Málaga distribution considering atmospheric attenuation and pointing errors. Applied Optics 57(19), 5505-5513 (2018). 